\title{
An Integrated Approach for Risk Assessment of Natural Disasters Using Cloud Computing
}

\author{
Plamena Zlateva, Yoshihiro Hirokawa, and Dimiter Velev
}

\begin{abstract}
The paper proposes an integrated approach for risk assessment of natural disasters using cloud computing, based on joint application of fuzzy logic models and an actuarial model. A hierarchical fuzzy logic system with several inputs and one output is designed. The risk assessment problem is defined as a multicriterial task that evaluates input variables (natural disaster indicators) and takes into account available information sources and the expert knowledge. The natural disaster indicators represent product of the probability of occurrence of a particular natural disaster and the aggregate loss caused by its action. The aggregate loss assessment is carried out by an actuarial model, more accurately, by the collective risk model. The main components of the collective risk model are discussed - the natural disaster magnitude (adverse events) and the negative consequences (severity of the damages). It is noted that the cloud computing is a model for providing on-demand Internet-based access to a shared pool of computing resources, including networks, storage and applications. The risk assessment results can support the state and local government to take more informed decisions regarding the efficient allocation of the available funding for the improvement of the population and infrastructure from natural disasters. The proposed fuzzy logic system with actuarial model is envisaged to be implemented as a part of an integrated information system for risk management of natural disasters in a cloud based environment. This system can be successfully used in e-government.
\end{abstract}

Index Terms-Actuarial model, cloud computing, fuzzy logic model, natural disasters, risk assessment.

\section{INTRODUCTION}

During the last decades the negative impact of natural disasters on human life, economy and environment is increased [1]. The annual losses resulting from floods, hurricanes, droughts, earthquakes, tornadoes, etc. cost billions of dollars. Natural disasters are difficult to avoid at all. Governments can propose strategies to mitigate and reduce the consequences only [2], [3].

The risk assessment of natural disasters is a major issue for the responsible risk management and the sustainable community development.

Hence there is a need to propose integrated approaches to risk assessment of natural disasters. The availability of an

Manuscript received March 27, 2013; revised May 9, 2013. This work was supported in part by the University of National and World Economy, Sofia, Bulgaria under Grant NI 1-8/2011.

P. Zlateva is with the ISER, Bulgarian Academy of Sciences, Sofia 1113, B1. 2, Bulgaria (e-mail:plamzlateva@abv.bg).

Y. Hirokawa is with the National Research Institute of Fire and Disaster, 4-35-3 Jindaiji-higashi-machi, Chofu, Tokyo, Japan (e-mail: hirokawa@fri.go.jp).

D. Velev is with the University of National and World Economy, Sofia 1700, Bulgaria (e-mail:dvelev@unwe.acad.bg). adequate assessment of the potential aggregate loss due to occurrence of adverse events would help taking more informed decisions for effective risk management [4]-[8].

On the other hand is well known that the cloud computing is a model for providing on-demand Internet-based access to a shared pool of computing resources, including networks, storage and applications [9].

The purpose of the paper is to propose an integrated approach for risk assessment of natural disasters using cloud computing, based on joint application of fuzzy logic models and an actuarial model. The actuarial model is based on the collective risk model. A hierarchical fuzzy logic system with several inputs, one output and some subsystems is designed.

The proposed model is envisaged to be implemented as a part of a Web information system for risk management of natural disasters using the Cloud Computing technology. This system can be successfully used in e-government.

\section{ESSENCE OF THE INTEGRATED APPROACH FOR RISK ASSESSMENT}

The essence of the integrated approach for risk Assessment is consists in joint application of fuzzy logic models and an actuarial model.

In this study, the fuzzy logic model is designed as a hierarchical system with several inputs and one output. The number of inputs corresponds to the indicators (linguistic variables), which describe the monitored natural disasters. The output represents a complex risk assessment. This hierarchical system consists of a few fuzzy logic subsystems.

An example of a three-level hierarchical fuzzy system with four inputs is shown in Fig. 1. The every level includes one fuzzy logic subsystem with two inputs. The output of the third fuzzy logic subsystem is output of the whole fuzzy system. This output variable gives the complex risk assessment relevant to the defined four input indicators. The value of the complex assessment is a criterion for decision making about the risk degree of the investigated objects (regions) from the monitored natural disasters. The higher output value corresponds to the higher risk degree [7].

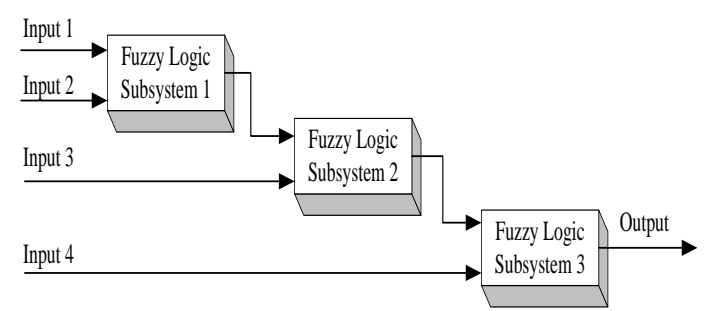

Fig. 1. A three-level hierarchical fuzzy system. 
Here, the every input indicator is represented as a product of the occurrence probability of a particular natural disaster and the aggregate loss caused by its action. The aggregate loss assessment is carried out by an actuarial model, more accurately, by the collective risk model.

The main components of the collective risk model are the natural disaster magnitude (adverse events) and the negative consequences (severity of the damages).

The collective risk model computes the aggregate loss as an independent sum of all losses (negative consequences caused by the various magnitudes of the natural disaster) from all magnitude natural disasters) incurred over a certain period [6]:

$$
S=X_{1}+X_{2}+\ldots+X_{N}
$$

where $S$ is the aggregate loss; $N$ - the number (frequency) of losses; $X_{i}$ - the severity of the $i$-th loss, for $i=1, \ldots, N$.

It is assumed that the loss severities $X_{i}, i=1, \ldots, N$ are independently and identically distributed (iid) as the loss severity random variable $X$. The loss-frequency $N$ is itself a nonnegative integer-valued random variable distributed independently of $X_{i}, i=1, \ldots, N$.

The aggregate loss $S$ is assumed to follow a nonnegative compound distribution. The loss-frequency random variable $N$ represents the primary distribution and the loss-severity random variable $X$ is secondary distribution of the compound distribution. Furthermore, $N$ and $X$ are assumed to be independent.

There are some advantages in modeling loss-frequency and loss-severity separately, and then combining them to determine the aggregate-loss distribution. Usually, primary and secondary distributions are determined by non-negative discrete random variables. In practice for the computation of the aggregate-loss distribution are used both recursive and approximate methods.

In the proposed approach for risk assessment of natural disasters, the every input indicator of the fuzzy logic system is defined by following function

$$
I_{j}=P_{j} \cdot C_{j}
$$

where $I_{j}$ is the value of the $j$-th input indicator, corresponding to the $j$-th natural disaster, $P_{j}$ - the occurrence probability of the $j$-th natural disaster, $C_{j}$ - the consequences (the aggregate loss) caused by action of the $j$-th natural disaster for $j=1, \ldots, m ; m-$ the number of the monitored natural disasters.

The consequences (the aggregate loss) caused by action of the $j$-th natural disaster are calculated by following collective risk model

$$
C_{j}=X_{1 j}+X_{2 j}+\ldots+X_{N j}
$$

where $N$ is the number of losses from the $j$-th natural disaster; $X_{i j}$ - the severity of the $i$-th loss from the $j$-th natural disaster with the $i$-th magnitude for $i=1, \ldots, N$.

\section{MAIN PROPERTIES OF COMPOUND Distributions}

A few main properties of compound distributions are described below [6], [8].

The moment generating function (mgf) of the random variable $X$ as a function of $t$ is denoted by $M_{X}(t)$. If the expectation exists then it is defined as follow

$$
M_{X}(t)=\mathrm{E}\left(e^{t X}\right)
$$

If the $M_{X}(t)$ exists for $t$ in an open interval around $t=0$, then the moments of $X$ exist and can be obtained by successively differentiating the $M_{X}(t)$ with respect to $t$ and evaluating the result at $t=0$.

The $r$-th derivative of the $M_{X}(t)$ is described by the following dependence:

$$
\begin{aligned}
M_{X}^{r}(t) & =\frac{d^{r} M_{X}(t)}{d t^{r}}=\frac{d^{r}}{d t^{r}} \mathrm{E}\left(e^{t X}\right)= \\
& =\mathrm{E}\left[\frac{d^{r}}{d t^{r}}\left(e^{t X}\right)\right]=\mathrm{E}\left(X^{r} e^{t X}\right) .
\end{aligned}
$$

Therefore, at the point $t=0$ for the $r$-th derivative of the $M_{X}(t)$ is obtained

$$
M_{X}^{r}(0)=\mathrm{E}\left(X^{r}\right)=\mu_{r}^{\prime}
$$

where by definition $r$-th initial moment of the random variable $X$ in the general case is given by the formula

$$
\mathrm{E}\left(X^{r}\right)=\sum_{i=1}^{\infty} x_{i}^{r} \cdot f_{X_{i}}\left(x_{i}\right)=\mu_{r}^{\prime}
$$

The probability generating function (pgf) of the nonnegative random variable $X$ is denoted by $\mathrm{P}_{X}(t)$. If the expectation exists then it is defined as follow

$$
\mathrm{P}_{X}(t)=\mathrm{E}\left(t^{x}\right)
$$

It is known that the mgf $M_{X}(t)$ and $\operatorname{pgf} \mathrm{P}_{X}(t)$ are related through the following equations

$$
M_{X}(t)=\mathrm{P}_{X}\left(e^{t}\right)
$$

The moment generating function (mgf) of the aggregate loss $S$ as a function of $t$ is denoted by $M_{S}(t)$.

If the primary distribution $N$ has $\operatorname{mgf} M_{N}(t)$ and the secondary distribution $X$ has $\operatorname{mgf} M_{X}(t)$, then the mgf of the compound distribution $S$ is deduce as follow 


$$
\begin{gathered}
M_{S}(t)=\mathrm{E}\left(e^{t S}\right)=\mathrm{E}\left(e^{t \cdot\left(X_{1}+X_{2}+\ldots+X_{N}\right)}\right)= \\
=\mathrm{E}\left(\mathrm{E}\left(e^{t . X_{1}+t \cdot X_{2}+\ldots+t \cdot X_{N}} \mid N\right)\right)=\mathrm{E}\left(\left(\mathrm{E}\left(e^{t . X}\right)\right)^{N}\right)= \\
=\mathrm{E}\left(\left(\mathrm{M}_{\mathrm{X}}(\mathrm{t})\right)^{N}\right)=\mathrm{E}\left(\left(\mathrm{e}^{\ln M_{\mathrm{X}}(\mathrm{t})}\right)^{N}\right)=M_{N}\left(\ln M_{X}(t)\right)
\end{gathered}
$$

If $N$ has $\operatorname{pgf} \mathrm{P}_{N}(t)$ and $X$ is nonnegative integer valued with $\operatorname{pgf} \mathrm{P}_{X}(t)$, then the pgf of $S$ is

$$
\begin{aligned}
& \mathrm{P}_{S}(t)=\mathrm{E}\left(t^{s}\right)=\mathrm{E}\left(\mathrm{E}\left(t^{X_{1}+X_{2}+\ldots+X_{N}}\right) \mid N\right)= \\
& =\mathrm{E}\left(\left(\mathrm{E}\left(t^{X}\right)\right)^{N}\right)=\mathrm{E}\left(\left(\mathrm{P}_{X}(t)\right)^{N}\right)=\mathrm{P}_{N}\left(\mathrm{P}_{X}(t)\right) .
\end{aligned}
$$

Furthermore, the aggregate loss $S$ is nonnegative and discrete, because the loss-severities take nonnegative discrete values. The mean of the aggregate loss $S$ is given by

$$
\begin{aligned}
\mathrm{E} S & =\mathrm{E}(\mathrm{E}(\mathrm{S} \mid \mathrm{N}))=\mathrm{E}(\mathrm{E}(N . X) \mid N)=\mathrm{E}(N . \mathrm{E}(X))= \\
& =\mathrm{E}\left(N . \mu_{X}\right)=\mu_{X} \mathrm{E}(N)=\mu_{N} \mu_{X}=\mathrm{E} N . \mathrm{E} X .
\end{aligned}
$$

The variance of the random variables $S$ is defined using the condition of independence between random variables $X$ and $N$, and the following relations:

$$
\begin{gathered}
\mathrm{D}(S)=\mathrm{E}\left(S^{2}\right)-(\mathrm{E} S)^{2} \quad \text { and } \quad \mathrm{E}\left(S^{2}\right)=\mathrm{E}\left(\mathrm{E}\left(S^{2} \mid N\right)\right) \\
\mathrm{E}(X \mid N)=\mathrm{E} X \quad \text { and } . \mathrm{D}(X \mid N)=\mathrm{D} X
\end{gathered}
$$

Thus the variance of the aggregate loss $S$ is is deduce as follow

$$
\begin{aligned}
\mathrm{D}(S)= & \mathrm{E}(\mathrm{D}(S \mid N))+\mathrm{E}\left((\mathrm{E}(S \mid N))^{2}\right)-(\mathrm{E}(\mathrm{E}(S \mid N)))^{2}= \\
& =\mathrm{E}(N \mathrm{D}(X \mid N))+\mathrm{D}(N \mathrm{E}(X \mid N))= \\
& =\mathrm{E}\left(N \cdot \sigma_{X}^{2}\right)+\mathrm{D}\left(N \cdot \mu_{X}\right)=\mu_{N} \cdot \sigma_{X}^{2}+\sigma_{N}^{2} \cdot \mu_{X}^{2} .
\end{aligned}
$$

The collective risk model is described only theoretically. Further research is needed particularly regarding its implementation for aggregate loss assessment of the critical infrastructure elements due to natural disasters for a given time interval.

\section{DESIGN OF FuZZY LOGIC MODEL}

The fuzzy logic models provide adequate processing of the expert knowledge and uncertain quantitative data [7]. The paper idea is to propose a fuzzy logic model for natural risk assessment the available information and the expert knowledge.
A typical fuzzy logic model for risk assessment from natural disasters as shown in Fig. 1.

In the example the natural disaster indicators are four, $m=4$. In particular, the fuzzy system inputs are defined as follow: Input 1 "Landslides" (landslips, creep); Input 2 "Mud-rock flows"; Input 3 "Floods"; Input 4 "Seismic hazard".

The inputs of the first fuzzy logic subsystem are Input 1 "Landslides" and Input 2 "Mud-rock flows", and the linguistic output variable is defined as Intermediate variable 1 "Gravitational processes".

The inputs of the second fuzzy logic subsystem are Intermediate variable 1 "Gravitational processes" and Input 3 "Seismic hazard", and the linguistic output variable is defined as Intermediate variable 2 "Geomorphological processes".

The inputs of the third fuzzy logic subsystem are Intermediate variable 2 "Geomorphological processes" and Input 4 "Floods". The output of the fuzzy subsystem is output of the whole fuzzy system.

In fuzzy logic system the input linguistic variables (four indicators and two intermediate) can be represented by three fuzzy membership functions: "Low", "Middle", and "High". The input variables can be assessed in the interval $[0,10]$ using trapezoid membership functions. The fuzzy logic system output (complex risk assessment) can be described by five fuzzy membership functions: "Very low", "Low", "Middle", "High", and "Very high". The complex natural risk can be assessed in the interval $[0,100]$ using triangular membership functions. The considered input and output membership functions are shown in Fig. 2.
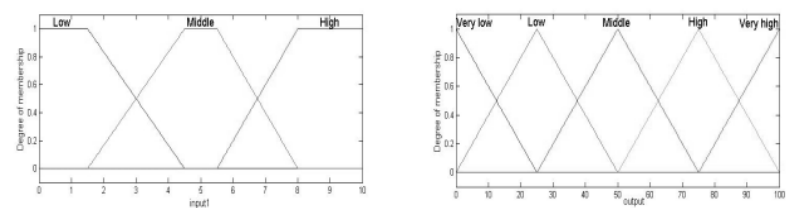

Fig. 2. Membership functions of the inputs and the system output.

The inference rules in the fuzzy logic system are defined through "IF - THEN"-clause.

In this example, the rule numbers of the knowledge base per each of the fuzzy logic subsystems are 9. Some of the inference rules are defined as follow:

IF "Landslides" is "High" and "Mud-rock" is "Middle" THEN "Gravitational processes risk" is "Middle";

IF "Gravitational processes risk" is "Low" and "Floods" is "Middle" THEN "Geomorphological

processes" is "Low";

IF "Gravitational processes risk" is "High" and "Floods" is "Middle” THEN "Geomorphological

processes" is "High";

IF "Geomorphological processes" is "Low" and "Seismic hazard" is "Low" THEN "Natural hazards" is "Very low";

IF "Geomorphological processes" is "Low" and "Seismic hazard" is "High" THEN "Natural hazards" is "Middle";

IF "Geomorphological processes" is "High" and "Seismic hazard" is "High" THEN "Natural hazards" is "Very high".

The fuzzy logic hierarchical system is designed in Matlab environment using Fuzzy Logic Toolbox and Simulink. The three fuzzy subsystems are built in the Mamdani type fuzzy 
inference system.

\section{AN ENVISAGE IMPLEMENTATION OF THE PROPOSED APPROACH IN A CLOUD ENVIRONMENT}

Research over the past several years has identified the independent evolution of four powerful forces - social, mobile, cloud and information [10]. As a result of consumerization and the ubiquity of connected smart devices, people's behavior has caused a convergence of these forces. Their existing architectures are becoming obsolete. The social media, cloud computing, mobile technology and large information volumes are converging to form a "nexus of forces" that will build upon and transform user behavior, creating business opportunities as it grows according to a report from Gartner IT research [11]. In the Nexus of Forces, information is the context for delivering enhanced social and mobile experiences. Mobile devices are a platform for effective social networking and new ways of work. Social links people to their work and each other in new and unexpected ways. Cloud enables delivery of information and functionality to users and systems. The forces of the Nexus are intertwined to create a user-driven ecosystem of modern computing [12].

Online social networking services and social media like Facebook, Twitter, Google+, etc. can act and try to solve many problems during natural disasters [13]. During disasters all the conventional communications generally stop functioning at this time interval while social media or networking services stay active. Online social networks allow the establishment of global relationships that are domain related or can be based on some need shared by the participants. There are large volumes of research work that have investigated whether or not the social network paradigm can be used to enable individuals and organizations to collaborate in mutually beneficial ways, in all stages of emergency management: mitigation, preparedness, response and recovery.

By the end of 2012 the mobile platform will become the dominant communications and interaction platform by best-practice organizations. The mobile platforms will allow the users of smartphones and tablet devices to have new capabilities at their disposssal. Tablets will become the virtual classroom, and an emerging class of tools will let people control and manage almost every aspect of their personal and professional life digitally.

Cloud computing is an on-demand service model for IT provision based on virtualization and distributed computing technologies. Cloud computing is a model for enabling ubiquitous, convenient, on-demand network access to a shared pool of configurable computing resources (e.g., networks, servers, storage, applications, and services) that can be rapidly provisioned and released with minimal management effort or service provider interaction. This cloud model is composed of five essential characteristics, three service models, and four deployment models [14]. Clouds are secure and though adaptation of authenticity, encryption, and meeting security software regulation large concern about secure can be put aside. The cloud allows for large amounts of computing power over short periods of time, like during a disaster, so relief agencies can respond to anything in the world. The cloud platform allows resources to be used on an elastic basis.

Organizations are looking for ways to find value in and insight from both structured and unstructured data from internal and external sources. This is expected to complement but not to replace long-standing information management programs and investments in data warehouses, business intelligence suites, reporting platforms and relational database experience. The concept of information known as Big data is not only managing large volumes of data, but also controlling the velocity and variety of data that exists nowadays. The ability to extract data from different sources to perform a specific task and the ability to provide information in real-time with the right context is essential. Information is stored everywhere. Social, mobile and cloud make information accessible, shareable and consumable at anytime and anywhere. The knowledge to capture the right information and utilize the smaller subsets applicable to a specific problem will be critical for avoiding risks.

Risk assessment as a service is a new paradigm for measuring risk as an autonomic method that follows the on-demand, automated, multitenant architecture of the cloud - a way to get a continuous risk score of the cloud environment with respect to a given tenant, a specific application or more generally, for use by new tenants and applications. Risk assessment provides a significant value in increasing trust in a commercial service, and thus appear particularly beneficial to the adoption of cloud computing. Traditional assessments developed for conventional IT environments do not readily fit the dynamic nature of the cloud [9].

The proposed approach is envisaged to be implemented as an instance of the "nexus of forces" paradigm in a cloud environment that allows:

- Using the computing cloud to rapidly converge geographically dispersed global experts at the start of an international incident, deploy a foundation of guidance in accordance with community leaders in a manner that empowers community members through education and smart technologies to support mitigation, response, recovery, and a resumption of societal normalcy at a level of functioning an order of magnitude higher than existed before.

- Using mobile communication devices for rapid threat/damage assessment of occurring events, as well as damage to critical infrastructure inland that necessitates mass sheltering of displaced community members.

- Using the power of nongovernmental organizations, rapidly responding government and corporate groups, social networking communities and other online social networks to locate and gather information, as well as to send help.

Cloud computing is the best solution to the needs and requirements of the government, organizations and individuals responding to catastrophic disasters. The availability, scalability, cost, speed of communication and potential security offer solutions to current dilemmas within the emergency response and relief work community are considered. Cloud computing services are more readily available for a response to a catastrophic event. Since the 
cloud applications are hosted at geographically dispersed locations, they are not at risk of going down if one of the facilities fails. Cloud computing is effective because they can scale when user load dramatically increases. The Clouds allow for flexibility since they can expand quickly as the application demands increase. Cloud computing provides the ability for users to communicate between those in the field with those coordinating efforts outside the field. With cloud computing if one has access to the Internet, whether though cell phone or a computer they can connect with the cloud.

\section{CONCLUSION}

The paper proposes an integrated approach for risk assessment of natural disasters based on joint application of fuzzy logic models and an actuarial model. A hierarchical fuzzy logic system with several inputs and one output is designed. The natural disaster indicators represent product of the probability of occurrence of a particular natural disaster and the aggregate loss caused by its action. The aggregate loss assessment is carried out by an actuarial model, more accurately, by the collective risk model. The proposed fuzzy logic system with actuarial model is envisaged to be implemented as a part of an integrated information system for risk management of natural disasters in a cloud based environment. This system can be successfully used in e-government.

\section{ACKNOWLEDGMENT}

The authors expresses his gratitude to the Science Fund of the University of National and World Economy, Sofia, Bulgaria for financial support under the Grant NI 1-8/2011, titled "Methodology for the Implementation of Web-based Integrated Information System for Risk Assessment Due to Natural Hazards".

\section{REFERENCES}

[1] J. Pollner, J. K. Watson, and S. Nieuwejaar, Disaster Risk Management and Climate Change Adaptation in Europe and Central Asia. Global Facility for Disaster Reduction and Recovery, The World Bank, 2010.

[2] J. Padli, M. Habibullah, and A. Baharom, "Economic impact of natural disasters' fatalities," International Journal of Social Economics, vol. 37 , no. 6 , pp. $429-441,2010$.

[3] Prevention Web - Strengthening climate change adaptation through effective disaster risk reduction. [Online]. Available: http://www.preventionweb.net.

[4] R. Kaas, M. Goovaerts, J. Dhaene, and M. Denuit, Modern ActuarialRisk Theory: Using R, New York: Springer-Verlag, 2008.

[5] S. Klugman, H. Panjer, and G. Willmot, Loss Models: From Data to Decisions (3rd ed.), New York: Wiley, 2008.

[6] Y. K. Tse, Nonlife Actuarial Models: Theory, Methods and Evaluation, Cambridge: Cambridge University Press, 2009.
[7] P. Zlateva, L. Pashova, K. Stoyanov, and D. Velev, "Fuzzy logic model for natural risk assessment in SW Bulgaria," pp. 109-113, vol. 13, IPCSIT, 2011.

[8] P. Zlateva and D. Velev, "An actuarial approach for aggregate loss assessment of the critical Infrastructure due to natural disasters," in International Proc. Economics Development and Research," pp. 24 1-345, vol. 30, Singapore: IACSIT Press, 2012.

[9] B. Kaliski and W. Pauley. (2010). Toward Risk Assessment as a Service in Cloud Environments. [Online]. Available: http://www.usenix.org/event/.

[10] D. Hinchcliffe. The "Big Five" IT trends of the next half decade: Mobile, social, cloud, consumerization, and big data. [Online]. Available: http://www.zdnet.com/blog/.

[11] Gartner. Gartner Says Nexus of Forces Social, Mobile Cloud and Information - Is the Basis of the Technology Platform of the Future [Online]. Available: http://www.gartner.com/it/page.jsp?id=2097215.

[12] Gartner. The Nexus of Forces: Social, Mobile, Cloud and Information. [Online]. Available: http://www.gartner.com/DisplayDocument?doc_cd=234840.

[13] A. Srikanth. Social media can solve many problems during natural disasters. [Online]. Available: http://infworm.com/

[14] The NIST Definition of Cloud Computing. [Online]. Available: http://csrc.nist.gov/publications/nistpubs/800-145/SP800-145.pdf.

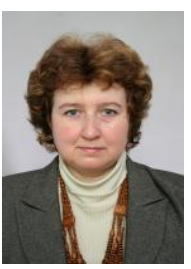

Plamena Zlateva is currently an associate professor at the Institute of System Engineering and Robotics at the Bulgarian Academy of Sciences, Sofia, Bulgaria. She hods M.Sc. degrees in Applied Mathematics from the Sofia Technical University and in Economics from the Sofia University St. Kl. Ohridski, and Ph.D. degree in Manufacturing Automation from the Institute of System Engineering and Robotics. Her main areas of academic and research interest are Control Theory, Mathematical Modeling and System Identification, Risk Theory, Risk Management.

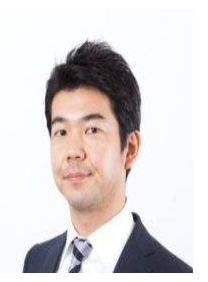

Yoshihiro Hirokawa is currently a research officer at the Research Planning Division of National Research Institute of Fire and Disaster (NRIFD). He holds Dr. Engineering in Mechanical Engineering from Tokyo Institute of Technology and $\mathrm{MSc}$ in Business Management from London Business School. His main areas of academic and research interest are Risk Management and Emergency Response against Natural Disasters, Earthquake Response Analysis, and Stress Measurement by Non-Destructive Testing.

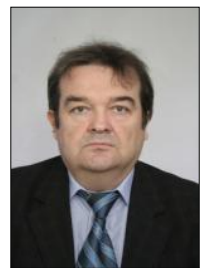

Dimiter Velev is a professor in the Department of Information Technologies and Communications at the University of National and World Economy, Sofia, Bulgaria. He holds M.Sc. degree in Electro- engineering from the Sofia Technical University, Bulgaria and Ph.D. degree in Engineering Sciences from the Institute of Modeling Problems in Power Engineering at the National Academy of Sciences of Ukraine, Kiev, Ukraine. His main areas of academic and research interest are Internet-Based Business Systems Modeling and Development, Service Oriented Architectures, Online Social Networks, Cloud Computing, Web Applications Development and Programming. His lectures cover such disciplines. 